\title{
Article
}

\section{Drones in B5G/6G Networks as Flying Base Stations}

\author{
Georgios Amponis ${ }^{1,2}$, Thomas Lagkas ${ }^{1, * \mathbb{D}}$, Maria Zevgara ${ }^{2}$, Georgios Katsikas ${ }^{3} \mathbb{D}$, Thanos Xirofotos ${ }^{3} \mathbb{D}$, \\ Ioannis Moscholios 4 (D) and Panagiotis Sarigiannidis 5 (D)
}

1 Department of Computer Science, International Hellenic University, 65404 Kavala, Greece; geaboni@cs.ihu.gr or gamponis@k3y.bg

2 K3Y Ltd., 1612 Sofia, Bulgaria; mzevgara@k3y.bg

3 UBITECH Ltd., 15231 Athens, Greece; gkatsikas@ubitech.eu (G.K.); txirofotos@ubitech.eu (T.X.)

4 Department Informatics \& Telecommunications, University Peloponnese, 22100 Tripolis, Greece; idm@uop.gr

5 Department of Electrical and Computer Engineering, University of Western Macedonia, 50100 Kozani, Greece; psarigiannidis@uowm.gr

* Correspondence: tlagkas@cs.ihu.gr

check for

updates

Citation: Amponis, G.; Lagkas, T.;

Zevgara, M.; Katsikas, G.; Xirofotos,

T.; Moscholios, I.; Sarigiannidis, P.

Drones in B5G/6G Networks as

Flying Base Stations. Drones 2022, 6,

39. https://doi.org/10.3390/

drones6020039

Academic Editors: Diego

González-Aguilera and Pablo

Rodríguez-Gonzálvez

Received: 15 December 2021

Accepted: 30 January 2022

Published: 5 February 2022

Publisher's Note: MDPI stays neutral with regard to jurisdictional claims in published maps and institutional affiliations.

Copyright: (C) 2022 by the authors. Licensee MDPI, Basel, Switzerland. This article is an open access article distributed under the terms and conditions of the Creative Commons Attribution (CC BY) license (https:/ / creativecommons.org/licenses/by/ $4.0 /)$.

\begin{abstract}
Advances in the fields of networking, broadband communications and demand for highfidelity low-latency last-mile communications have rendered as-efficient-as-possible relaying methods more necessary than ever. This paper investigates the possibility of the utilization of cellular-enabled drones as aerial base stations in next-generation cellular networks. Flying ad hoc networks (FANETs) acting as clusters of deployable relays for the on-demand extension of broadband connectivity constitute a promising scenario in the domain of next-generation high-availability communications. Matters of mobility, handover efficiency, energy availability, optimal positioning and node localization as well as respective multi-objective optimizations are discussed in detail, with their core ideas defining the structure of the work at hand. This paper examines improvements to the existing cellular network core to support novel use-cases and lower the operation costs of diverse ad hoc deployments.
\end{abstract}

Keywords: cellular-enabled drones; aerial base stations; 5G/B5G/6G cellular networks

\section{Introduction}

Next-generation cellular communications constitute a key enabler of the greater adoption of next-generation Internet of Things (NG-IoT)-based technologies, by allowing an increase in the number of interconnected orders of magnitude, offering high data rates and near real-time responsiveness as well as addressing various requirements of NG-IoT [1]. Elevated security, better quality of service (QoS), reduced end-to-end delay and higher datarates are directly correlated to the utilization of higher frequencies, which in turn demand more power and introduce additional dependencies and overhead at the hardware and software levels. The aforementioned parameters, requirements and considerations significantly limit the available spectrum of competent low-power devices and introduce power, effort and networking overhead, especially in ad hoc and remote sensing applications. Millimeter wave (mmWave) communications, multiple-input-multiple-output (MIMO) and non-orthogonal multiple access (NOMA) are some examples of the technological novelties introduced by $5 \mathrm{G}$ and highlighted in the $6 \mathrm{G}$ standard currently under development. Novel orchestration mechanisms specific to next-generation cellular networks allow for and push towards a more intelligent edge, with an increasing number of functionalities being implemented in an ad hoc, distributed manner.

It is important to note that $5 \mathrm{G}$ and $6 \mathrm{G}$ introduce the requirement for a previously unseen densification of networks. This is particularly challenging and constitutes a challenge directly addressable via aerial and ad hoc communications. FANETs have the potential of bringing about this technological revolution by means of the intelligent relaying and provision of broadband in otherwise isolated areas and cut-off hubs. Individual UAVs can constitute aerial base stations as a means of serving local wireless networks, e.g., wireless 
sensor networks (WSNs). Correspondingly, networks of flying base stations constitute great candidates for the units responsible for the opportunistic interconnection of isolated nodes and hubs by employing context-aware routing and swarm topology formation in a 3D aerial grid. The necessary performance optimizations of such networks are in need of scalable and decentralized architectural approaches.

The ongoing development and wide adoption of $5 \mathrm{G}$ have driven the design of wireless systems beyond 5G (B5G), including the sixth generation (6G). This new generation of cellular communications should be able to unlock the full potential of the numerous autonomous services that encompass both past and emerging trends. More specifically, 6G should bring novel, breakthrough wireless technologies and innovative network architectures into focus.

The sixth generation of cellular communications has the potential to offer extreme data rates to address the massive connectivity aspect and enable an extremely high throughput, even under extreme conditions or in emergency scenarios in which node density, spectrum and infrastructure availability, and traffic patterns may vary. Additionally, B5G/6G networks will be pivotal in achieving a high degree of immersion and capacity whilst also offering a uniform and highly deterministic quality of experience, required by novel applications. A key application area of next generation networks is delivering real-time feedback-based services enabled by near-zero latency to fulfill the requirements of said novel applications.

As highlighted in [2], airborne communication base stations are envisaged to constitute a pivotal component of the B5G/6G cellular architecture, as mentioned due to their flexible and highly mobile nature. As networks and UEs become increasingly mobile, it is absurd to keep gNBs and relaying equipment solely statically, especially for providing coverage, e.g., in hotspots and in areas with sub-optimal infrastructure (in environments recovering from disaster, rural/suburban areas that suffer due to lack of financial incentives for network operators, etc.).

The first large-scale attempt by the industry was made by Google, aiming to address lack of infrastructure to provide Internet access for the currently non-covered population. Project Loon aspired to provide connectivity in remote or rural areas using stratosphere balloons. These balloons would hover at a height of approximately $20 \mathrm{~km}$. The core idea revolved around using wind waves blowing in the right direction to steer the balloon to the area in need of coverage, and establishing a stratosphere-layer mobile ad hoc network [3]. Nevertheless, high operational costs rendered this idea non-viable in the commercial landscape, and the project was shut down. Similarly, in recent years, there has been a substantial amount of research in the domain of satellite-enabled broadband provision. The authors in [4] reviewed the potential of the usage of satellites for the provision of 5G NR channels. The authors investigated issues arising from a severe Doppler shift and impact on reception and demodulation on a user-equipment level. Several severe limitations render satellite-enabled NR channels unpractical for mobile or real-time applications. Dedicated receivers and demodulation hardware are required, along with software and hardware methods to mitigate issues related to the random-access process.

Increased ease in establishing a direct LOS link with ground users and other cellular infrastructures can support B5G/6G networks in formulating more reliable links and offering a wider coverage area, immune to reflection-induced or environmental losses. These advantages of airborne BSs combined with the requirements set by the oncoming 6G networks have led to the investigation of a spectrum of drone-enabled and cellular-specific communication networks, namely air-to-ground channel characteristics, the optimal positioning of drones (either as sole relays or parts of a swarm), as well as flight trajectory optimizations.

An important and rather novel improvement of $6 \mathrm{G}$ is the direct incorporation of artificial intelligence (AI) in the network core as a means of supporting seamless data-centric and context-aware services capable of great degrees of self-optimization [5]. Additionally, 6G will enable the modern cellular communications landscape to meet the desired high levels of end-to-end reliability and correspondingly low end-to-end latency to support 
ultra-high mobility scenarios, such as flying vehicles. B5G and 6G communications are envisaged to support wireless federated learning (WFL) [6] through the joint optimization of resources targeting a reduction in the delay for novel federated applications [7]. Inherent support for AI within the network core will be pivotal in supporting novel applications in a spectrum of domains, with a great example being drone-enabled intelligent surveillance and machine vision-supported remote sensing [8].

B5G/6G, a major driving force behind the vision of $6 \mathrm{G}$, involves the deployment of connected and autonomous vehicle systems (CAVs) and drone communications. Research efforts in the field of CAV and drone-based communication systems have been steadily increasing in both academia and industry, targeting strict requirements, especially ultra-low latency and unprecedented communication reliability. As the industry is shifting towards wireless, real-time and high-throughput networking, drone base stations are envisaged to constitute pivotal assets.

Table 1 showcases the main differences between 5G and 6G networks and the main improvements with regard to their core attributes [9].

Table 1. Comparison of 5G and 6G attributes.

\begin{tabular}{lll}
\hline Attribute & 5G & 6G \\
\hline Peak Frequency & $110 \mathrm{GHz}$ (W-band) & $10 \mathrm{THz}$ \\
\hline Peak Spectral Efficiency & $30 \mathrm{bps} / \mathrm{Hz}$ & $100 \mathrm{bps} / \mathrm{Hz}$ \\
\hline Peak Data-rate & $20 \mathrm{Gbps}$ & $1000 \mathrm{Gbps}$ \\
\hline End-to-End Latency & $10 \mathrm{~ms}$ & $1 \mathrm{~ms}$ \\
\hline Connection Density & $1 \mathrm{million}$ per sq. kilometer & $10 \mathrm{million}$ per sq. kilometer \\
\hline Supported Node Mobility & $500 \mathrm{~km} / \mathrm{h}$ & $1000 \mathrm{~km} / \mathrm{h}$ \\
\hline
\end{tabular}

Table 2 compares the present work to already existing surveys' drone-BS related papers. The present work is focused not only on surveying and reviewing the current state of the art, but also documenting what is missing from the current research landscape. This paper also contributes to identifying the challenges directly associated with the NR landscape, examining the usability of aerially supported communication frameworks, offered benefits, the implications and challenges of such technological leaps, mainly revolving around resource allocation and power consumption, node mobility and path formation, positioning, security and offered QoS. Our approach proves to be the most complete in terms of the variables considered for the survey comparison.

By reading the presented work, the reader will have gained applicable knowledge in the domain of next-generation ad hoc communications, as well as the capability to critically compare and review related literature, thus supporting further research in this rapidly evolving field. Furthermore, the aerially supported applications of 5G communications are disseminated, and implications of secure, resource-aware and intelligent orchestration are examined. As the presented work constitutes an output of the 5G-INDUCE H2020 project, parallels are drawn-where applicable-between the examined use cases and challenges.

The layout of the rest of this paper is as follows: Section 2 follows this introduction, which documents the evolution of cellular communications and the potential offered by 5G/B5G/6G networks; Section 3 introduces the reader to the concept of using unmanned aerial vehicles to extend cellular connectivity and is divided into two main subsections: Section 3.1, which describes the various potential use cases of this technology and Section 3.2, which analyzes the main challenges currently faced. Section 4 discusses the overall developments enabling the acceleration of aerially supported next-generation cellular communications. Section 5 concludes this paper with comments regarding further work in this field and possible extensions of existing research. Figure 1 provides a high-level view of the paper's structure, discussed topics and overall flow. 
Table 2. Related research.

Consideration and/or Analysis of:

\begin{tabular}{lllllll} 
Related Work & Drone-BSs & 5G/B5G/6G & Energy Availability & Path Planning & BS Positioning Drone-BS Use Cases \\
\hline Nikooroo et. al. $\checkmark$ & $\checkmark$ & $\checkmark$ & - & $\checkmark$ & - \\
\hline Mach et. al. & $\checkmark$ & - & $\checkmark$ & - & $\checkmark$ & - \\
\hline Plachy et. al. & $\checkmark$ & - & - & - & - & - \\
\hline Zhao et. al. & $\checkmark$ & - & - & - & - & $\checkmark$ \\
\hline Fotouhi et. al. & $\checkmark$ & - & - & - & - & - \\
\hline Becvar et. al. & $\checkmark$ & - & $\checkmark$ & - & $\checkmark$ & - \\
\hline Bayerlein et. al. $\checkmark$ & - & - & $\checkmark$ & $\checkmark$ & - \\
\hline Zhang et. al. & $\checkmark$ & - & - & - & $\checkmark$ & - \\
\hline Alzenad et. al. $\checkmark \checkmark$ & - & $\checkmark$ & - & - & $\checkmark$ \\
\hline Bushnaq et. al. $\checkmark$ & - & - & - & $\checkmark$ \\
\hline Mozaffari et. al. $\checkmark$ & $\checkmark$ & - & $\checkmark$ & $\checkmark$ \\
\hline Our work & $\checkmark$ & $\checkmark$ & $\checkmark$ & $\checkmark$ & \\
\hline
\end{tabular}
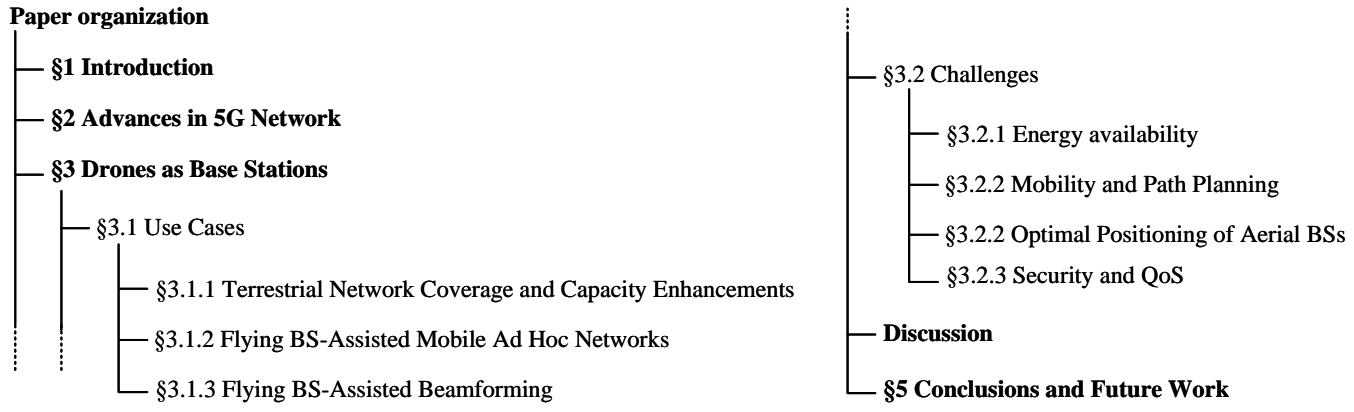

Figure 1. High-level structure of the presented work.

\section{Advances in 5G Networks}

The core elements of the 3GPP 5G architecture are defined in ETSI TS 123501 V15.2.0 (2018-06). As demonstrated in Figure 2, the core 5G services (implemented in the form of network functions (NFs)), are the network slice selection function (NSSF), the network exposure function (NEF), the network repository function (NRF), the policy control function $(\mathrm{PCF})$, the unified data management (UDM), the application function (AF), the authentication server function (AUSF), the access and mobility management function (AMF), the session management function (SMF), the user plane function (UPF), the data network (DN), the radio access network (RAN), and the user equipment (UE).

In the context of the presented work, the most important components can be narrowed down to the AMF, the RAN and the UE. The AMF is responsible for registration and connection management, as well as ensuring reachability and managing UE mobility. As demonstrated in Figure 1, the supported mobility for $5 \mathrm{G}$ networks reaches up to $500 \mathrm{~km} / \mathrm{h}$, and up to $100 \mathrm{~km} / \mathrm{h}$ for the upcoming $6 \mathrm{G}$ networks. Handling node mobility is enabled by this network function. The RAN utilizes radio transceivers (gNodeB/gNB instances) to facilitate cellular connectivity; gNBs provide the New Radio (NR) user plane and control plane protocol interfaces with the UE. According to 3GPP, a device utilized by an end-user to facilitate communication with another user or service is a UE, which is in turn connected to the gNB. 


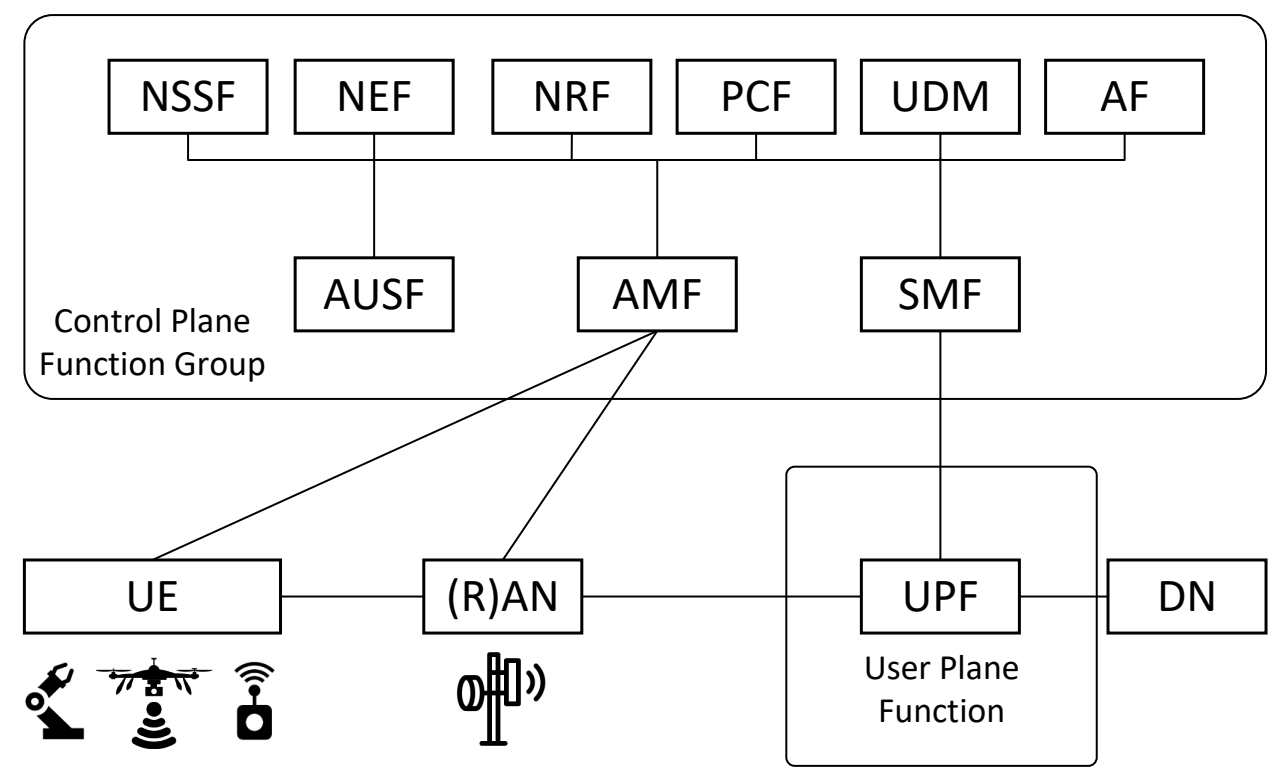

Figure 2. 3GPP-compliant 5G architecture.

It can be assumed that drones have a dual function in this architecture. On the one hand, drones constitute consumers of the services offered by the 5G core (5GC) and can be considered as the devices with the end-user is in direct interface with, while on the other hand, drones utilized as flying base stations are implementing the services offered by the gNBs as they offer the end-users a connection to the 5GC and the respective $\mathrm{NF}$ services. Since drones are typically not in a direct interface with the rest of the $5 \mathrm{G}$ core network architecture, they can be better described as UEs implementing (part of) the gNB services to serve other nodes in terms of communication enhancements and range extension for both terrestrial and mobile ad hoc networks. The authors in [10] considered the possibility of using low-cost solutions to realize a flying 5G UPF, to assist the attachment of mobile devices to the network core, whose functionality is also implemented within the drone itself. This method promises to enable easier offloading. However, this method poses several security risks, as it exposes core network services to end-users and potential attackers. As mentioned, due to the presented architecture being highly compartmentalized, a high degree of distribution can be achieved. This directly supports the dockerization and offloading of NFs and tasks via resource-aware orchestrators, thus enabling traditionally non-cellular infrastructure (namely drones or other edge devices) to implement functionalities of the core cellular network.

Existing technologies fall short in terms of fully leveraging smart 5G infrastructure capabilities. Even the most mature orchestration technologies to date are typically restricted to serve rather simple $5 \mathrm{G}$ slicing requests, resulting in:

1. Partial or no support for advanced services, namely the use-cases mentioned in the presented paper and an advanced slicing mechanism associated with application offloading;

2. The inability to discover and directly expose the entire range of smart 5G infrastructure capabilities, which may be advertised by an underlying network function virtualization orchestrator (NFVO) platform.

The intelligent OSS of the 5G-INDUCE project, is of particular interest to the currently examined set of applications, as it will be capable of exposing the core network capabilities to the end-users at a high application level without revealing any potentially confidential low-level (infrastructure-related) information. This is particularly valuable for usage in the networks established in an ad hoc or on-demand manner. By offering novel intelligent orchestration services, 5G-INDUCE aims to support drone-assisted network performance 
and coverage monitoring for critical scenarios. The main goal of the 5G-INDUCE project is thus to enable an entirely new spectrum of ad hoc and high-fidelity services.

\section{Drones as Base Stations}

As highlighted in [11], drones are relatively low-cost solutions that can efficiently extend next-generation connectivity in areas that are normally either unreachable via traditional infrastructure or momentarily unreachable due to, e.g., network disruptions. Similarly, drone base stations (BSs) can increase the quality of a connection between an ad hoc/remote node and a given cellular base station. Drone BS are also attractive solutions for providing reliable, broadband and wide-area temporary wireless connectivity during special events or harsh scenarios, namely natural disasters [12], smart farming [13], sporting events, and many more scenarios where the permanent installment of cellular infrastructure is unnecessary and comes with considerable monetary overhead.

The authors in [14] stated that, in addition to the above, drone BSs at high altitudes are expected to provide long-term and cost-effective connectivity for rural areas. The integration of drone BSs with other physical layer techniques such as $\mathrm{mmW}$ and massive MIMO as well as cognitive radios is a promising solution for providing data-intensive services and is expected to create new challenges for next-generation flying. The optimal positioning of drone BSs is one of the critical challenges to be overcome in dense deployment scenarios. For that purpose, the optimal positioning of the drone BSs is an issue in need of tackling. Optimal positioning is one of the most critical challenges and must be addressed in dense B5G/6G deployment scenarios.

The utilization of drones as airborne base stations enables a service provider to offer enhancements of connectivity and capacity of already existing terrestrial wireless networks, with cellular ones being the main areas of interest. Compared to conventional terrestrial base stations, the advantage of using UAVs as flying base stations is their ability to dynamically readjust their altitude, and their comparative ease in terms of establishing direct LOS links to terrestrial gNBs or UE instances. Due to their inherent characteristics in terms of mobility and flexible altitude adaptation, cellular-enabled drone base stations can effectively support existing cellular systems by providing additional communication capacity to areas and ensuring network coverage in difficult-to-reach rural areas, as such deployments are naturally three-dimensional and offer unrestricted mobility. There exist numerous types of UAVs to facilitate connectivity and undertake the role of a base station, with each having its advantages and disadvantages in terms of mobility, autonomy, maneuverability and maximum payload. Table 3 compares the main types of available UAVs.

Table 3. Comparison of UAV types.

\begin{tabular}{lllll}
\hline UAV Type & $\begin{array}{l}\text { Stationary Flight } \\
\text { (Yes/No) }\end{array}$ & $\begin{array}{l}\text { Typical Battery Life } \\
\text { (mins) }\end{array}$ & $\begin{array}{l}\text { Typical Velocity } \\
(\mathbf{m} / \mathbf{s})\end{array}$ & $\begin{array}{l}\text { Typical Payload } \\
(\mathbf{k g})\end{array}$ \\
\hline Multi-rotor & yes & $=<15$ & $=<11$ & $=<2.5$ \\
\hline Fixed-wing & no & $=<60$ & $=<22$ & $=<14$ \\
\hline Baloon & yes & $=<60$ & $=<2.5$ & $=<4.5$ \\
\hline
\end{tabular}

\subsection{Use Cases}

The researchers in [15] noted that mobile 5G is a key driver of network services in different industries, and therefore accelerates the digital transformation of the respective services. $5 \mathrm{G}$ provides more advanced and enhanced capabilities compared to $4 \mathrm{G}$, and following the same pattern, 6G will introduce the entire industry to a new era. New industry-specific standards for next-generation cellular networks are already being incorporated in 3GPP Releases, with 3GPP Release 15 defining new radio (NR) and Packet Core evolution as a means of establishing fully interoperable deployments. The next 3GPP Release (Release 16) aspires to support gigahertz cellular communications as prescribed by IMT-2020, whilst 
also incorporating new communication standards for smart cars and Industry 4.0 factories. Considering the aforementioned remarks, airborne BSs will mostly be used for relaying information and facilitating range extension where cellular infrastructure fails to deliver. The most prominent use-cases for drones functioning as base stations are:

- The extension of terrestrial network coverage and capacity;

- The assistance of mobile ad hoc networks (MANETs);

- $\quad$ Beamforming applications.

\subsubsection{Terrestrial Network Coverage and Capacity Enhancements}

The first drone-enabled base station scenario revolves around terrestrial cellular network enhancements. In this scenario, it is assumed that cellular-enabled drones function as service providers; aerial nodes functioning as cellular base stations can provide pivotal improvements to ultra-dense small cell networks, which are highlighted in B5G/6G nextgeneration communications. Regarding next-generation $\mathrm{mmW}$ communications, droneenabled flying base stations find great applicability in establishing short-term line-of-sight (LOS) links among gNBs and UEs. Thus, the coverage and capacity of wireless networks can be effectively enhanced, whilst next-generation communications can be supported in a more effective manner, especially in dense cells. Additionally, as mentioned in Section 3, MIMO-based techniques have the potential to formulate an entirely new and dynamically reconfigurable enhanced cellular network, capable of providing never-seen-before high capacity services. A great example of on-demand terrestrial network coverage and capacity enhancements is the establishment of high-throughput links in first-response and emergency scenarios, in which the existing infrastructure is either damaged or inadequate; in this case, aerial base stations can be used to alleviate the load on the terrestrial cellular grid, or provide broadband connectivity where no infrastructure was available in the first place. Figure 3 showcases a possible application of on-demand terrestrial network coverage enhancement in the case of a wildfire. In this scenario, the line-of-sight between the radio antenna (gNB) and the corresponding UEs associated with the end user in need (firefighter) is blocked. Temporarily deploying a drone BS helps alleviate this issue by introducing a new path, thereby establishing a temporary yet direct and reliable link between the end user and cellular infrastructure.

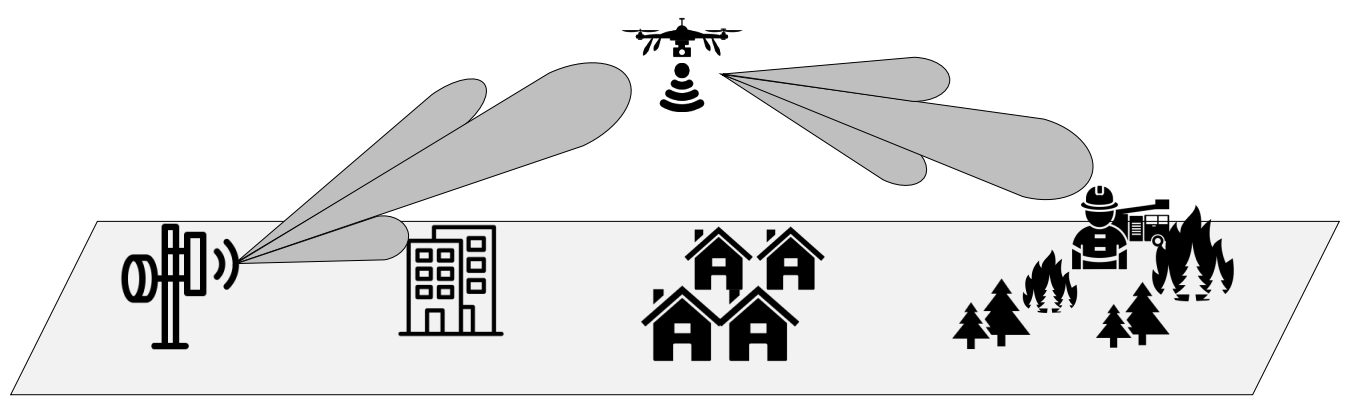

Figure 3. Terrestrial network coverage enhancement: a drone BS-supported firefighting scenario.

Moreover, network enhancements find substantial applicability in the formation of reliable communication links in rural and/or remote areas, with little-to-no existing infrastructure; this scenario supports the rejuvenation of rural areas as well as smart farming, and can assist in endeavors to close the digital gap. The use of aerial base stations is a promising solution to a number of challenges associated with terrestrial IoT networks as well, namely wireless sensor networks (e.g., ad hoc WSNs). Drone BSs can be deployed to provide reliable and energy-efficient uplink and downlink for device-to-device IoT communications, due to drones being effectively deployed in a manner aimed at reducing the shadowing and blockage effects, which constitute major causes of signal attenuation and losses in wireless links. Connectivity enhancements and terrestrial network capacity increases cannot be achieved without sufficiently optimizing the positioning of all communicating nodes in the 
3D space. The efficient positioning of drones is thus of the utmost significance in terms of connecting immobile UEs throughout the course of the connectivity-supporting process. This is particularly true in the case of low-power IoT devices, which require their respective gateways to be placed on locations such that the aforementioned devices can successfully establish a connection to the cellular network using the minimum required transmission power [16]. In this manner, drone BSs can support massive B5G/6G IoT diverse networks by constantly updating their relative positions to ensure optimal connectivity and reduce the need for permanent cellular infrastructure installations.

\subsubsection{Flying BS-Assisted Mobile Ad Hoc Networks}

Given their mobility and LOS-establishing capabilities, UAVs can support mobile ad hoc networks (MANETs) on the terrestrial plane, and more specifically vehicular ad hoc networks (VANETs). With the advent of smart and self-driving cars, the requirement for constant, uninterrupted and real-time communication is more pressing than ever. FANETs and singular UAVs have repeatedly proven their usability in terms of supporting deviceto-device communications, and given the increased support for high mobility in B5G/6G networks (see Table 1), they are excellent candidates for facilitating real-time information exchange and message broadcasting among mobile networked peers. A good example of this type of communications is UAV-enabled safety-related information broadcasting across numerous vehicles without a direct LOS or sufficient network coverage [17]. Aerial BSs can also enhance the reliability of device-to-device and inter-vehicular links by mitigating issues caused by interferences, which are a result of an increased number of re-transmissions. Figure 4 demonstrates an inter-vehicular communication scenario supported by drone-BSs.

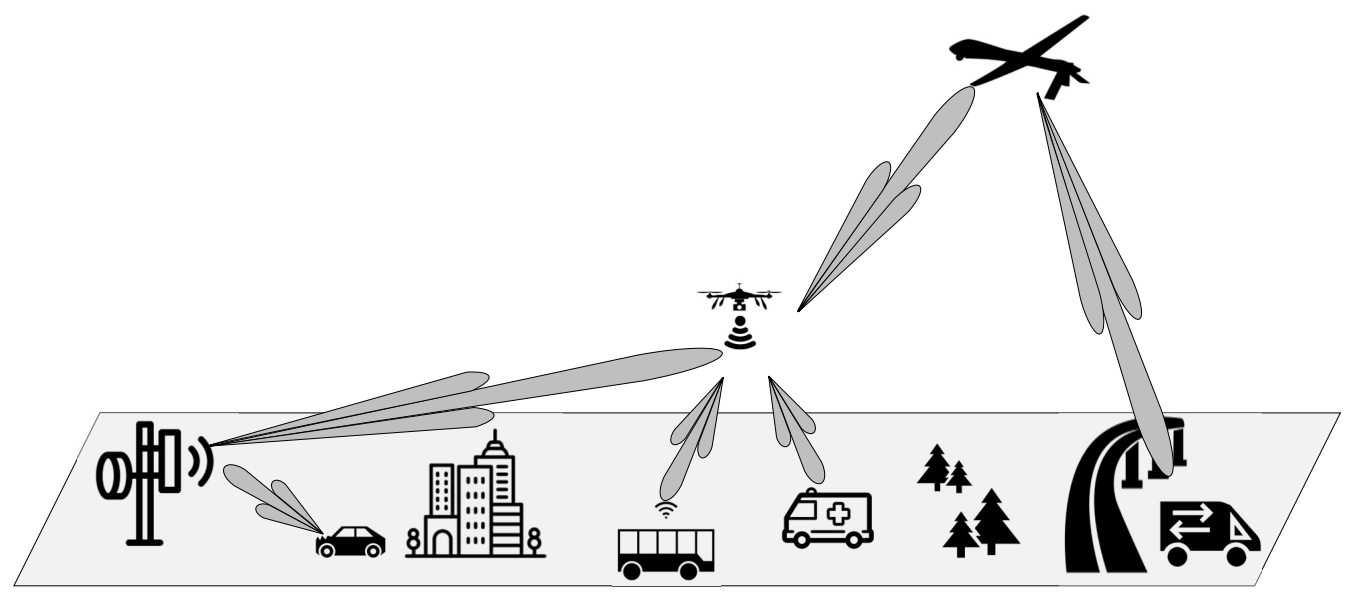

Figure 4. Drone-BS-assisted vehicular communications scenario.

Furthermore, airborne BSs offer a new non-terrestrially confined spectrum of diverse networking opportunities to boost reliability and last-mile connectivity in the networks at hand. An intelligent approach to accommodating mobile ad hoc networking is the clustering of ground UE instances into mobile swarms. This can be implemented to enable drone BSs to potentially communicate with a singular networked UE instance functioning as a "representative" (cluster head) of the mobile terrestrial cluster; the cluster head is responsible for distributing messages via broadcasting, and respectively, providing gateway services to the rest of the cluster nodes. Following this approach, the connectivity of mobile terrestrial ad hoc networks can be significantly improved by adopting clusterbased approaches and leveraging the unique mobility characteristics of drones. To that end, as is the case with the terrestrial network enhancement use-case scenario, drones will significantly increase the quality and reliability of their offered services by considering efficient placement and swarm nodes' relative positioning, where applicable. The matter of optimal positioning and path planning are discussed in detail in Section 3.2.3. 


\subsubsection{Flying BS-Assisted Beamforming}

In this scenario, drones can be viewed as airborne antenna elements, that can be conjointly utilized for performing massive MIMO, 3D MIMO and $\mathrm{mmW}$ communications. Beamforming using aerial elements can be pivotal in reducing intercell interference by forming distinct beams to simultaneously cover desired grid elements.

Research in recent years has been focused on full-dimension MIMO communications, where the horizontal and vertical dimensions of a cellular network alike, are utilized for beamforming. This supports the formulation of distinct beams in the cellular network, and can be resorted to as a means of minimizing intercell interference, which is projected to be a major issue in $6 \mathrm{G}$ communications. Three-dimensional beamforming solutions show great potential in offering significantly higher system throughput, all whilst supporting a greater number of UE instances, effectively tackling issues associated with high network density in, e.g., urban or industrial environments, which are key targeted use-cases for next-generation cellular networks. The aforementioned improvements render the evolution of MIMO communications from 2D to 3D not only beneficial but potentially mandatory for the wide applicability of B5G/6G communications. As highlighted in [18], cellular-enabled aerial BSs can be key enablers of high-density cellular networks by supporting intercell interference mitigation and management by beamforming the horizontal and vertical channel planes. Figure 5 showcases the potential for intercell interference mitigation for leveraging drone BSs, thus paving the path towards high-density next-generation cellular networks.

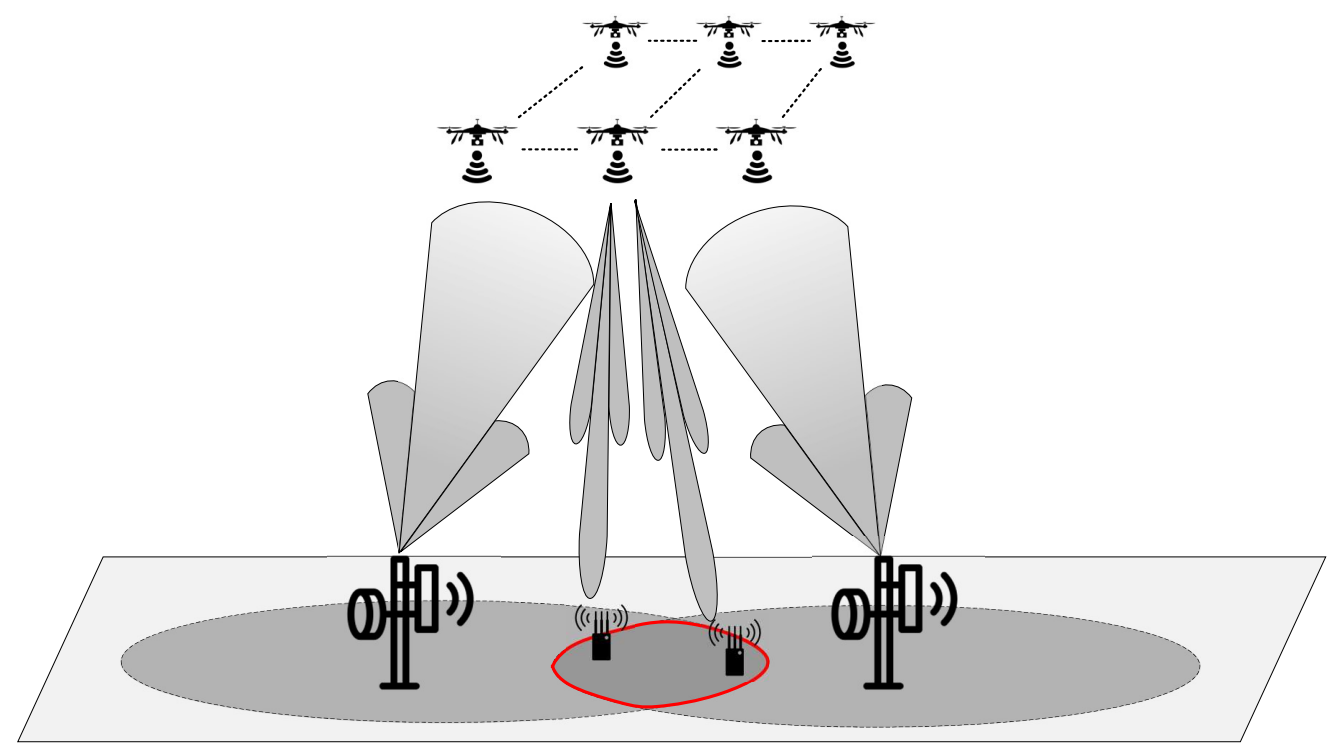

Figure 5. Beamforming: a drone BS-supported intercell interference mitigation scenario.

Drone-supported 3D MIMO is more suitable for high-density scenarios, and even more so in cases in which UE instances are distributed across a three-dimensional grid with different elevation angles (assuming a direct LOS with the respective cellular base station). As aerial base stations are typically significantly elevated in comparison to terrestrial UE, their respective altitude and elevation angles' differences can be relatively easily distinguished and considered as routing, relaying and dynamic antenna re-positioning metrics. Additionally, as elevation differences easily support direct LOS conditions, aerial base stations enable efficient and effective beamforming in a three-dimensional grid. When compared to conventional (terrestrially deployed and static) antenna array systems, a drone-based antenna array has the following advantages:

- The number of antenna elements is not limited by spatial constraints;

- Beamforming gain can be increased on-demand by adjusting array element (drone) spacing;

- Drones' mobility allows for effective beam-steering in virtually any 3D direction; 
- Utilization of drone swarms within an array formation can provide the capability to form a massive antenna array which can virtually accommodate any arbitrary shape and perform beamforming;

- $\quad$ Energy optimization or tethering (discussed in Section 3.2.1) can increase reliability to near terrestrial-node levels.

The authors in [19] engaged in a thorough analysis of the potential usage of drone swarm-based scattering for adaptive beamforming to efficiently relay data streams to locations normally not inside the transmitting node's range of communication or LOS. The proposed swarm-based beamforming methodology revolves around a ground-based transmitter radiating towards a swarm of drones, where each "antenna element" (individual drone) carries a half-wavelength resonant wire functioning as a scattering object, which in turn works as a reflector antenna radiating the BS's transmitted signal. The researchers investigated potential methods for optimizing the process of adjusting the altitude of drones, and as such, the phase of the scattered field from each array element to enable support for the formulation of desired radiation patterns. A strong argument for the usage of quadcopters as beamforming elements is the fact that because all signal phase shifting is implemented by adjusting the drone locations, no excessive, computationally intense or additional phase-shifting circuitry is required on-board. The researchers' optimization algorithm was able to form a beam composed of two main lobes, with a low level of side-lobes, which could be steered in the desired direction for different patterns.

Similarly, the researchers in [20] attempted to produce a directional beam to increase a network's quality of service, more specifically concerning cellular downlink. The novelty of their approach stems from the fact that the authors aimed to maximize coverage whilst considering human body-induced losses and model respective optimization procedures. The researchers used a uniform linear array of antennas at the transmitter and designed the optimized beam direction to maximize the number of covered users while considering the QoS constraint in the network. The significance of the authors' work stems from the fact that their developments are centered around the usage of $\mathrm{mmW}$ communication frameworks while considering the channel blockage effects of medium-to-large bodies; this enables the facilitation of optimizations aware of the environmental parameters and characterizing the respective link.

\subsection{Challenges}

This subsection is dedicated to the detailed analysis of the main identified challenges hindering the wider adoption of aerially enabled cellular base stations. The identified challenges can be narrowed down two five main issues, namely energy availability, mobility and path planning, positioning of nodes, security and privacy issues, and the offered quality of service. As the nature of the interfaces among the relaying equipment and the next-generation cellular network core is highly compartmentalized (assuming an ETSI TS 123501 V15.2.0-compliant 5G architecture), drone BSs belong to the RAN layer as UE instances, as highlighted in Section 3. All challenges, especially security and quality of service, are affected by this compartmentalized, "blackbox" approach, as the 5G core has no authoritative access to the radio-layer.

\subsubsection{Energy Availability}

An important issue with drone-based BSs is energy availability. As relays in B5G and 6G cellular networks are expected to relay greater volumes of information and provide reliable and sufficient QoS, throughput and minimal latency, the energy expenditure of relays rises exponentially. The energy expenditure of UAVs functioning as relays is mainly associated with:

- $\quad$ Energy consumed for the purpose of flying and hovering above a desired location;

- $\quad$ Energy consumed for communication and on-board processing;

A substantial amount of research has gone into designing energy-efficient routing and communication schemes to prolong battery lifespan via the usage of, e.g., received signal 
strength indication (RSSI) and the drone position data [21]. However, since the propulsion energy is significantly more than the payload energy, energy-efficient communication will not highly affect the flight time, which is of utmost importance for establishing a network of aerial cellular base stations. It can be safely assumed that for the purpose of enabling long flight times, battery technology has to keep up with the newly introduced requirements set by $\mathrm{B} 5 \mathrm{G} / 6 \mathrm{G}$ communications.

In all cases, the goal of energy-aware optimizations in regard to BS communication and mobility metrics is to minimize transmission power, whilst considering a predefined constraint for the minimum data rate which UEs require in all cases [22]. This type of optimization balances energy availability and the offered QoS. Energy consumption is in turn constrained by either maintaining at the same coverage area, or maximizing; in turn, QoS is constrained by either maintaining its current value or maximizing it.

In that spirit, the authors in [2] proposed a tether-based UAV setup for the deployment of drones as aerial base stations in 6G networks and engaged in an analysis of the mobilityendurance trade-off. The methodology proposed in the context of tethering $5 \mathrm{G} / 6 \mathrm{G}$ enabled quadcopters solves the inherent limitations of the short flight time and reliable backhaul links. The ground station supplies the airborne BS with energy whilst also providing the data link via a psychical tether. It is safely concluded that the most important defining factor for the offered QoS and overall functionality is the placement of individual drone relays. This is especially true for tethered UAVs, as they have substantial limitations in terms of horizontal mobility, thus reducing reachable positions in the 3D space. The authors in [23] analyzed hybrid drone-specific power supply systems that combine batteries with other types of energy sources, namely fuel cells, solar cells, supercapacitors, tethering and laser-enabled in-flight recharging. The aforementioned technologies are envisaged to help alleviate performance and autonomy issues, thus bringing about a drone-supported next-generation cellular landscape.

In the context of eliminating unnecessary energy expenditure, the authors in [22] proposed a solution for the elimination of the redundant movement of aerial BSs. The energy-saving framework they proposed relies on deriving new positions for the base stations considering the overall mobility and movement patterns of UEs. The authors considered that there exist UE mobility scenarios, in which it is not necessary to reposition the base station, mainly due to slight QoS variations falling within an acceptable spectrum. This multi-objective optimization problem can be summarized as "reducing globally-spent BS hovering energy, whilst maintaining networking capacity approximating the one associated with optimal node positions". It is suggested, that for maximizing energy availability of non-tethered (free-flying drone BSs), it is suggested that the aerial nodes do not precisely follow the best QoS-enabling position, but rather stay in a pre-computed distance from the optimum. Following this non-QoS-centric approach, the energy consumed for the purpose of flying can be kept to a minimum; this is implemented without sacrificing mobility, but establishing a threshold of acceptability for the loss of QoS.

As mentioned earlier, tethering techniques can be resorted to in order to remove battery-life constraints. The researchers in [24] proposed a new drone-based mobile relaying system, in which a laser beacon is employed to wirelessly charge the energy-constrained UAV relay. This approach of preserving energy and increasing flight time is of great interest, as it can potentially help solve the dilemma of choosing between mobility and energy availability. The authors aimed to conjointly maintain a global optimum, considering both required transmission power and mobility-specific parameters. The authors proposed two algorithms to solve this optimization problem, and showed that the laser beacon wavelength and environmental parameters such as weather conditions greatly impact both data and power transmission efficiency.

\subsubsection{Mobility and Path Planning}

In light of the aforementioned remarks, it can be assumed that node mobility capabilities and swarm-wide path planning is of utmost importance for the effective relaying of 
cellular communications for all three examined scenarios (terrestrial and mobile network coverage and capacity enhancements and beamforming applications) in Section 3.1. The mobility-awareness and optimal positioning of aerial base stations is an intricate and rather convoluted problem [25], with its complexity being a direct function of an entire spectrum of highly volatile parameters, namely:

- $\quad$ The number of aerial BSs participating in the relaying;

- The type of interfaces among these participating nodes (inter-drone relaying, conjoint formation of array antennas);

- $\quad$ The elevation, angle, position and velocity of each node relative to the respective gNB;

- $\quad$ Energy availability, expected energy expenditure and estimated uptime for new links;

- $\quad$ The topology of the terrain and potential blockages in LOS.

The researchers in [26] developed "SEDMAG", a 6G-specific path-planning algorithm for usage with aerial IoT nodes in both single-node and swarm deployments, mainly revolving around monitoring and extensible to cellular-relaying and WSN data harvesting scenarios. The authors' work was envisioned to reduce localization latency as well as energy overhead, by trading off precision in localization; this renders the SEDMAG algorithm useful for non-beamforming-related scenarios, where accuracy and localization precision are of utmost importance for phase shifting. Using a smart search algorithm and graph reduction, the researchers managed to provide better positioning efficiency when compared to non-dynamic path planning algorithms, namely Zcurve, but also dynamic path planning algorithms such as DREAMS in terms of errors and energy consumption. In line with resource-aware orchestration in next-generation cellular networks, the authors proposed a smart load-balancing approach capable of balancing drones load in swarm deployment scenarios and resulting in the reduction in localization delay. The method of function for SEDMAG and its respective derivatives is dividing the area of interest into equal smaller areas and assigning an aerial node to each said smaller area. The SEDMAG algorithm is tasked with positioning the drone(s) so that all areas of interest are adequately covered; it then computes the shortest path for the drone to visit all areas required to be covered by the network and decides on the order in which they are to be visited. The SSEDMAG algorithm (a derivative of SEDMAG) utilizes an intelligent searching approach to shorten the drone's flight path. Similarly, SSEDMAG-reduced aims to further reduce the drone's trajectory by re-applying SSEDMAG on the reduced flight path. Lastly, the SSEDMAG-reduced-balanced algorithm is the one finding the greatest applicability in swarm deployment scenarios, as it is tasked with balancing the tasks and overall load among drones, aiming to achieve a global localization optimum for sufficient coverage given a minimum number of trajectory shifting and path alterations.

\subsubsection{Optimal Positioning}

Researchers in [27] analyzed the optimal positioning of UAV BSs in conjunction with transmission power allocation, user clustering and next-generation NOMA networks. The authors attempted to elicit a means of optimally clustering UEs and positioning drone BSs so that only a minimum required transmission power is utilized for successful link establishment, whilst ensuring that QoS is above a given minimum threshold at all times. The proposed multi-objective optimization method allows for a significant increase in the duration of aerial coverage of a given terrestrial area. Overall, the proposed solution can be divided into three distinct sub-tasks:

- Task 1: Select the optimal clusters of a given number of UEs to be simultaneously served by a NOMA network;

- $\quad$ Task 2: Allocating the optimal transmission power to each node;

- Task 3: Determining the position of the flying BS in the 3D space.

Correspondingly, the identified constraints are:

- The available propulsion energy;

- The guaranteed minimum capacity for each mobile user. 
Finally, the direct optimization goal is to increase the duration of communication coverage in NOMA. The authors measured an increase in the order of $67-270 \%$ when compared to existing solutions, assuming a constant propulsion power.

The authors in [28] considered the scenario of connectivity enhancement in the case of on-demand ad hoc deployments of cellular networks. To that end, the researchers considered the optimal positioning of drones in swarm deployments so that they can mitigate interferences and offer on-demand communication-extending services to a substantial amount of UEs. It is important to note the highly dynamic and volatile nature of the networking requirements of end-users in such ad hoc deployments. The authors of [28] focused on the issue of maximizing the offered QoS (and consequently user satisfaction), by proposing an algorithm capable of:

- $\quad$ Associating UEs with the best-suited aerial BS;

- $\quad$ Finding optimal positions of all aerial BSs.

Additionally, the authors considered the performance of the genetic and particle swarm optimization algorithms, which are evaluated in terms of performance, accuracy and offered QoS (calculated as available data rates). The authors showed that the particle swarm optimization algorithm is substantially less complex compared to the genetic algorithm, while the latter one is more efficient in its utilization of nodes. The tradeoff seems to be: time complexity vs. efficacy.

The researchers in [29] considered the usage of multiple drone-mounted radio heads as a means of providing on-demand connectivity and dynamic cloud radio access networking capabilities. To facilitate this, the authors realized an optimal drone positioning mechanism to address the requirement for transmission power minimization. The researchers divided the multi-objective optimization problem into two distinct ones, one per each axis of movement:

- Horizontal positioning of drones (minimization of distance sum);

- Vertical positioning of drones (maximization of coverage).

For the first problem (horizontal positioning optimization), the authors resorted to the Weiszfeld algorithm to compute and output the point that minimizes the total distance to be covered by the sum of (re)transmissions [30]. Similarly, the second problem (vertical positioning optimization) was approached with the goal of calculating the optimal elevation angle and the terrestrially projected radius of the coverage area per drone. The resulting algorithm proved to be capable of offering the minimum required transmission power while maintaining a pre-defined acceptable performance threshold in terms of end-user connectivity.

\subsubsection{Security and QoS}

NG communications inevitably give rise to various security concerns. As a substantially greater and ever-increasing amount of data is being relayed per unit of time, the infiltration of a NG network is automatically rendered more rewarding. Furthermore, the advent of novel time-sensitive use cases are exponentially increasing reliance on lowlatency and near real-time communications; this implies that network stability becomes mandatory, and QoS must be kept above a certain threshold at all times. Should a network service be interrupted, various negative cascading events would take place, especially considering smart vehicles and generally VANET-supporting NG services, as shown in Section 3.1.2.

Security and QoS in next-generation cellular networks are closely correlated, as they are associated with the interfaces established between relaying nodes and the cellular core (namely the $5 \mathrm{G}$ core). The usage of drones as means of connectivity extension poses a considerable security threat. As such devices constitute 5G/B5G user equipment instances, they belong to the RAN layer of the $5 \mathrm{G}$ architecture. This means that security features implemented on a 5G-core level have little to no authority with regard to handling UE authorization, security and QoS provision. Drones such as UEs are directly exposed 
(both physically and from a networking point of view) to end users. A potential security breach in the RAN-exposed NFs is a serious security rise, as it can escalate rather quickly. Authentication among the networked drone BSs is even more challenging to implement in a reliable manner [31]. More specifically, the following 5G components and network functions are exposed to the UE:

- $\quad \mathrm{gNB}$, the terrestrial cellular base station (RAN);

- $\quad \mathrm{AMF}$, the mobility management function of (core network).

It becomes evident that the creation of an abstraction layer to secure QoS and security is mandatory for a sustainable and reliable transition to aerially supported high-density cellular networks. This abstraction layer comprises additional security functions and orchestration mechanisms that improve the architecture of cellular communications [32]. In this domain, the 5G-INDUCE project aims to facilitate the incorporation of additional features for security, and cognitive capabilities that cannot be supported by existing environments to ensure QoS above an end-user-defined threshold. Special focus is given to QoS assurance in conjunction with security, by leveraging the smart exposure of application-specific interfaces to end-users. With throughput reaching the scales of dozens of Gbps, end-to-end latency being reduced to the scale of a few milliseconds, and network availability being stretched to its limit, monitoring link- and network-layer metrics to accommodate a targeted QoS is extremely challenging to do while preserving privacy, security and keeping computational overhead to a minimum.

\section{Discussion}

In the presented work, we discussed matters of $5 \mathrm{G}$ and B5G/6G communications in a spectrum of scenarios, resource utilization, various types of optimizations and the elicitation of efficient, energy-aware and QoS-preserving methods of providing high-quality services to a set of UEs. We identified the three main scenarios which are expected to significantly benefit from drones in next-generation cellular networks functioning as flying base stations.

To this end, the 5G-INDUCE project is envisaged to offer a variety of services and components as add-ons to the 5G core architecture presented in Section 2 and described on a high-level (considering interfaces among the management and network orchestration (MANO) and the virtual/physical infrastructure and the NFVO) in Figure 6. 5GINDUCE offers a full-stack NetApp management platform to orchestrate services and functionalities, mainly in the industrial domain. Orchestration enhancement can support data confidentiality, securely encrypt critical infrastructure management and monitoring, and reliable operator-drone communication interfaces. The scenarios described in Sections 3.1.1 and 3.1.2 and (to a lesser degree) Section 3.1.3, strongly relate to the targeted NetApps of the 5G-INDUCE project and are aligned with its goal of establishing easily extensible yet secure and QoS-aware next-generation cellular connectivity in critical scenarios. All aforementioned use-cases rely on novel orchestration algorithms for the deployment of services over containerized realms.

The challenges currently faced by the entire research and industrial landscape range from security and privacy, to licensing and AI-related issues, namely explainability and legislative/ethical concerns in regard to automated piloting, no-fly-zones and potential collisions; all these areas require more research in the near future, as little to no work has targeted the aforementioned parameters in a networking context. It can be easily deduced that the complexity of said issues will increase, even more so with the increase in network heterogeneity and the additional requirements entailed. Low-earth orbit satellites (and constellations thereof) also seem to be a rather promising technology in terms of supporting ubiquitous connectivity for NR networks. With this in mind, future protocols, frameworks, and even hardware modulators and demodulators shall be designed to support satellite-to-drone connectivity where applicable. Consequently, standardization for all developments targeting the aforementioned challenges is going to be a direct focus of all relative standardization groups and institutes in the coming years. 
Said challenges are to be addressed through the upcoming cellular networks' services' ability to compartmentalize all their components and to only expose the desired entrypoints to the end-users. This can be managed by combining application orchestration with network services orchestration mechanisms, so as to allow the higher layer to manage the deployment and lifecycle of the services at hand, and the lower orchestration layer to optimally interact with the network and computational resources of the nodes. The upcoming compartmentalized orchestration mechanisms will thus have to build on existing knowledge and go beyond the ETSI NFV MANO, following the respective 3GPP specifications, while being aligned to the expected 3GPP Release 16 standards, as mentioned in Section 3.1. Overall, the extension of connectivity and the provision of services on-demand seems to be pivotal in the formulation of novel core orchestrator architectures. All the scenarios described in this paper strongly rely on the establishment of good-quality and secure links between gNBs and UE instances for effectively relaying and seamless coordination among them, especially in the case of beamforming. Thus, future research must be as focused on service-provision optimizations as it must be focused on improving the existing 5G core service-based architecture.

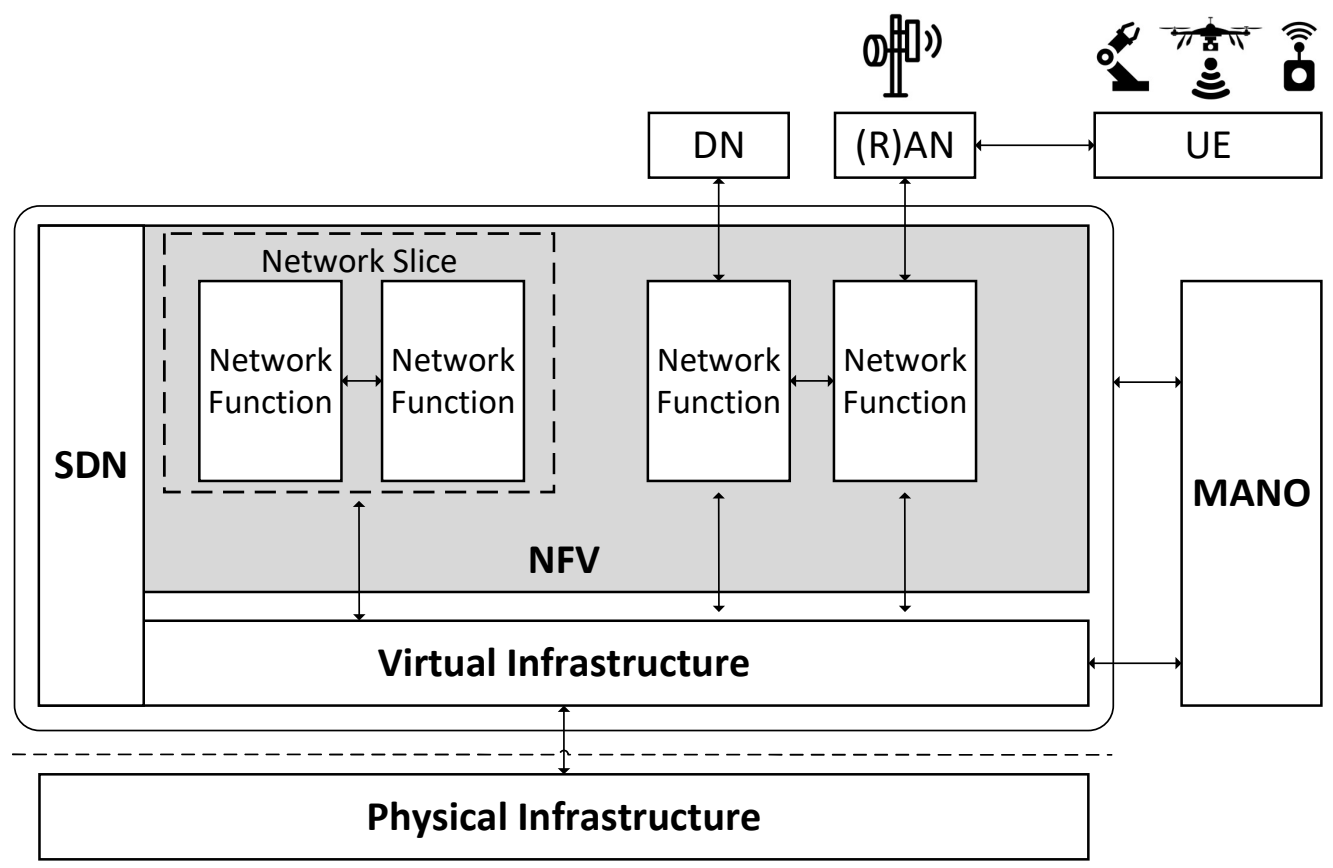

Figure 6. High-level overview of the virtual-physical infrastructure compartmentalization and key component interfaces.

\section{Conclusions and Future Work}

This paper discussed a number of potential use-cases for drone base stations in B5G/6G networks and the implications of their usage in various environments. Matters of resource allocation, optimal positioning and channel provision are discussed in detail, in order to establish a common discussion ground for future developments in regard to cellular connectivity enhancements. Moreover, this paper has discussed improvements in $5 \mathrm{G}$ orchestration mechanisms as a means of achieving substantial improvements in terms of throughput, task allocation optimization mechanisms and swarm positioning optimizations. Given ongoing developments in cross-layer metric utilization for task and resource orchestration, future developments can potentially revolve around the environment-aware predictive deployment of drone base stations using machine learning, as has been already proposed in [33]. On a technical level, the formulation of multi-objective optimization problems in the near future will be capable of accommodating and considering various weights and newly introduced realistic constraints to deduce the optimal solution in a machine learning-enabled manner; this will significantly boost developments in the domain of path 
planning and optimal positioning, as discussed in Section 3.2.3. Advances in orchestration and network function compartmentalization will support efforts in complementing the terrestrial cellular system in the face of adverse events to offload excess traffic or increase cellular range.

Most of the developments in the context of the usage of B5G/6G-enabled drones as base-stations can be expressed as direct functions of localization and optimal relative positioning efficacy. As routing algorithms increase in efficiency, aerial ad hoc deployments will be capable of formulating three-dimensional grids capable of offering more efficient relaying services. This use case scenario is discussed in detail by the authors in [34]. More specifically, the authors discussed how an efficient drone base-station 3D placement algorithm will support efforts to maximize the total number of UE instances whilst utilizing the minimum required power. Resource-aware routing is an ongoing development in the research community, with a relative study of resource-aware cross-layer routing for FANETs performed by the authors of the present paper in [35]. Cross-layering (the utilization of, e.g., physical/MAC-layer parameters in the context of network-layer routing) has the potential to increase performance for the physical and link-layer processing, which will, in turn, enable lower costs for operators in terms of extremely dense deployments namely in Industry 4.0 use-cases [36] or emergency applications [37]. Finally, the seamless mobility and integration of heterogeneous links for collaboration in the same ad hoc cellular network, will be supported by multi-connectivity and a cell-less architecture envisaged in [38] and enabled the utilization of novel scheduling algorithms and a new core network design. Regarding the optimization of the resource allocation and positioning of aerial nodes, in future work, the problem of optimal bandwidth allocation should be considered, along with the issue of accommodating highly mobile UEs, as well as the effective mitigation of interference among BS nodes and securing the underlying cellular infrastructure by means of high degrees of compartmentalization and the strategic exposure of network resources to UEs.

Author Contributions: Conceptualization, T.L., P.S., G.K. and G.A.; methodology, G.A., T.L. and I.M.; software, G.A. and T.L.; validation, T.L., G.A. and P.S.; formal analysis, G.A., T.L. and P.S.; investigation, G.A., T.L., M.Z., G.K., T.X., I.M., P.S.; resources, T.L., P.S. and T.X.; data curation, T.L., I.M. and G.K.; writing—original draft preparation, G.A. and T.L.; writing—review and editing, T.L., G.A. and M.Z.; visualization, G.A.; supervision, T.L. and P.S.; project administration, T.L. and P.S. All authors have read and agreed to the published version of the manuscript.

Funding: The project leading to this application has received funding from the European Union's Horizon 2020 research and innovation programme under grant agreement No 101016941.

Institutional Review Board Statement: Not applicable.

Informed Consent Statement: Not applicable.

Data Availability Statement: No new data were created or analyzed in this study. Data sharing is not applicable to this article.

Conflicts of Interest: The authors declare no conflict of interest.

\section{References}

1. Pliatsios, D.; Goudos, S.K.; Lagkas, T.; Argyriou, V.; Boulogeorgos, A.A.A.; Sarigiannidis, P. Drone-Base-Station for NextGeneration Internet-of-Things: A Comparison of Swarm Intelligence Approaches. IEEE Open J. Antennas Propag. 2022, 3, $32-47$. [CrossRef]

2. Kishk, M.; Bader, A.; Alouini, M.S. Aerial Base Station Deployment in 6G Cellular Networks Using Tethered Drones: The Mobility and Endurance Tradeoff. IEEE Veh. Technol. Mag. 2020, 15, 103-111. [CrossRef]

3. Kaur, S.; Randhawa, S. Google LOON: Balloon-powered internet for everyone. AIP Conf. Proc. 2018, 2034, 020006. [CrossRef]

4. Saarnisaari, H.; de Lima, C.M. 5G NR over Satellite Links: Evaluation of Synchronization and Random Access Processes. In Proceedings of the 2019 21st International Conference on Transparent Optical Networks (ICTON), Angerrs, France, 9-13 July 2019; pp. 1-4. [CrossRef] 
5. $\quad$ Rekkas, V.P.; Sotiroudis, S.; Sarigiannidis, P.; Karagiannidis, G.K.; Goudos, S.K. Unsupervised Machine Learning in 6G Networks -State-of-the-art and Future Trends. In Proceedings of the 2021 10th International Conference on Modern Circuits and Systems Technologies (MOCAST), Thessaloniki, Greece, 5-7 July 2021; pp. 1-4. [CrossRef]

6. Bouzinis, P.S.; Diamantoulakis, P.D.; Karagiannidis, G.K. Wireless Federated Learning (WFL) for 6G Networks ${ }^{4}$ Part I: Research Challenges and Future Trends. IEEE Commun. Lett. 2022, 26, 3-7. [CrossRef]

7. Bouzinis, P.S.; Diamantoulakis, P.D.; Karagiannidis, G.K. Wireless Federated Learning (WFL) for 6G Networks-Part II: The Compute-Then-Transmit NOMA Paradigm. IEEE Commun. Lett. 2022, 26, 8-12. [CrossRef]

8. Chaschatzis, C.; Karaiskou, C.; Mouratidis, E.G.; Karagiannis, E.; Sarigiannidis, P.G. Detection and Characterization of Stressed Sweet Cherry Tissues Using Machine Learning. Drones 2022, 6, 3. [CrossRef]

9. Rekkas, V.P.; Sotiroudis, S.; Sarigiannidis, P.; Wan, S.; Karagiannidis, G.K.; Goudos, S.K. Machine Learning in Beyond 5G/6G Networks-State-of-the-Art and Future Trends. Electronics 2021, 10, 2786. [CrossRef]

10. Fattore, U.; Liebsch, M.; Bernardos, C.J. UPFlight: An enabler for Avionic MEC in a drone-extended 5G mobile network. In Proceedings of the 2020 IEEE 91st Vehicular Technology Conference (VTC2020-Spring), Antwerp, Belgium, 25-28 May 2020; pp. 1-7. [CrossRef]

11. Bariah, L.; Mohjazi, L.; Muhaidat, S.; Sofotasios, P.C.; Kurt, G.K.; Yanikomeroglu, H.; Dobre, O.A. A Prospective Look: Key Enabling Technologies, Applications and Open Research Topics in 6G Networks. IEEE Access 2020, 8, 174792-174820. [CrossRef]

12. Selim, M.Y.; Kamal, A.E. Post-Disaster 4G/5G Network Rehabilitation Using Drones: Solving Battery and Backhaul Issues. In Proceedings of the 2018 IEEE Globecom Workshops (GC Wkshps), Abu Dhabi, United Arab Emirates, 9-13 December 2018; pp. 1-6. [CrossRef]

13. Livanos, G.; Ramnalis, D.; Polychronos, V.; Balomenou, P.; Sarigiannidis, P.; Kakamoukas, G.; Karamitsou, T.; Angelidis, P.; Zervakis, M. Extraction of Reflectance Maps for Smart Farming Applications Using Unmanned Aerial Vehicles. In Proceedings of the 2020 12th International Symposium on Communication Systems, Networks and Digital Signal Processing (CSNDSP), Porto, Portugal, 20-22 July 2020; pp. 1-6. [CrossRef]

14. Mozaffari, M.; Saad, W.; Bennis, M.; Nam, Y.H.; Debbah, M. A Tutorial on UAVs for Wireless Networks: Applications, Challenges, and Open Problems. IEEE Commun. Surv. Tutorials 2019, 21, 2334-2360. [CrossRef]

15. Slalmi, A.; Chaibi, H.; Chehri, A.; Saadane, R.; Jeon, G. Toward 6G: Understanding network requirements and key performance indicators. Trans. Emerg. Telecommun. Technol. 2021, 32, e4201. [CrossRef]

16. Saym, M.M.; Mahbub, M.; Ahmed, F. Coverage Maximization by Optimal Positioning and Transmission Planning for UAVAssisted Wireless Communications. In Proceedings of the 2021 International Conference on Science Contemporary Technologies (ICSCT), Dhaka, Bangladesh, 5-7 August 2021; pp. 1-4. [CrossRef]

17. Su, Y.; LiWang, M.; Hosseinalipour, S.; Huang, L.; Dai, H. Optimal Position Planning of UAV Relays in UAV-assisted Vehicular Networks. In Proceedings of the ICC 2021-IEEE International Conference on Communications, Virtual, 14-23 June 2021; pp. 1-6. [CrossRef]

18. Seifi, N.; Zhang, J.; Heath, R.; Svensson, T.; Coldrey, M. Coordinated 3D Beamforming for Interference Management in Cellular Networks. Wirel. Commun. IEEE Trans. 2014, 13, 5396-5410. [CrossRef]

19. Egarguin, N.J.A.; Jackson, D.R.; Onofrei, D.; Leclerc, J.; Becker, A. Adaptive Beamforming Using Scattering From a Drone Swarm. In Proceedings of the 2020 IEEE Texas Symposium on Wireless and Microwave Circuits and Systems (WMCS), Waco, TX, USA, 26-28 May 2020; pp. 1-6. [CrossRef]

20. Vaezy, H.; Salehi Heydar Abad, M.; Ercetin, O.; Yanikomeroglu, H.; Omidi, M.J.; Naghsh, M.M. Beamforming for Maximal Coverage in mmWave Drones: A Reinforcement Learning Approach. IEEE Commun. Lett. 2020, 24, 1033-1037. [CrossRef]

21. Spyridis, Y.; Lagkas, T.; Sarigiannidis, P.; Argyriou, V.; Sarigiannidis, A.; Eleftherakis, G.; Zhang, J. Towards 6G IoT: Tracing Mobile Sensor Nodes with Deep Learning Clustering in UAV Networks. Sensors 2021, 21, 3936. [CrossRef] [PubMed]

22. Becvar, Z.; Mach, P.; Nikooroo, M. Reducing Energy Consumed by Repositioning of Flying Base Stations Serving Mobile Users In Proceedings of the 2020 IEEE Wireless Communications and Networking Conference (WCNC), Seoul, Korea, 25-28 May 2020; pp. 1-7. [CrossRef]

23. Boukoberine, M.N.; Zhou, Z.; Benbouzid, M. Power Supply Architectures for Drones-A Review. In Proceedings of the IECON 2019-45th Annual Conference of the IEEE Industrial Electronics Society, Lisbon, Portugal, 14-17 October 2019; Volume 1, pp. 5826-5831. [CrossRef]

24. Zhao, M.M.; Shi, Q.; Zhao, M.J. Efficiency Maximization for UAV-Enabled Mobile Relaying Systems With Laser Charging. IEEE Trans. Wirel. Commun. 2020, 19, 3257-3272. [CrossRef]

25. Pijnappel, T.R.; van den Berg, J.L.; Borst, S.C.; Litjens, R. Drone-Assisted Cellular Networks: Optimal Positioning and Load Management. In Proceedings of the 2021 IEEE 93rd Vehicular Technology Conference (VTC2021-Spring), Helsinki, Finland, 25-28 April 2021; pp. 1-6. [CrossRef]

26. Kouroshnezhad, S.; Peiravi, A.; Haghighi, M.S.; Jolfaei, A. Energy-Efficient Drone Trajectory Planning for the Localization of 6G-Enabled IoT Devices. IEEE Internet Things J. 2021, 8, 5202-5210. [CrossRef]

27. Nikooroo, M.; Becvar, Z. Optimal Positioning of Flying Base Stations and Transmission Power Allocation in NOMA Networks. IEEE Trans. Wirel. Commun. 2021, 1. [CrossRef]

28. Plachy, J.; Becvar, Z.; Mach, P.; Marik, R.; Vondra, M. Joint Positioning of Flying Base Stations and Association of Users: Evolutionary-Based Approach. IEEE Access 2019, 7, 11454-11463. [CrossRef] 
29. Pliatsios, D.; Sarigiannidis, P.; Goudos, S.K.; Psannis, K. 3D Placement of Drone-Mounted Remote Radio Head for Minimum Transmission Power Under Connectivity Constraints. IEEE Access 2020, 8, 200338-200350. [CrossRef]

30. Pliatsios, D.; Sarigiannidis, P.; Moscholios, I.D.; Tsiakalos, A. Cost-efficient Remote Radio Head Deployment in 5G Networks Under Minimum Capacity Requirements. In Proceedings of the 2019 Panhellenic Conference on Electronics Telecommunications (PACET), Volos, Greece, 8-9 November 2019; pp. 1-4. [CrossRef]

31. Feng, C.; Liu, B.; Guo, Z.; Yu, K.; Qin, Z.; Choo, K.K.R. Blockchain-based Cross-domain Authentication for Intelligent 5G-enabled Internet of Drones. IEEE Internet Things J. 2021, 1. [CrossRef]

32. Odarchenko, R.; Usik, P.; Volkov, O.; Simakhin, V.; Gospodarchuk, O.; Burmak, Y. 5G Networks Cyberincidents Monitoring System for Drone Communications. In Proceedings of the 2019 IEEE 5th International Conference Actual Problems of Unmanned Aerial Vehicles Developments (APUAVD), Kiev, Ukraine, 22-24 October 2019; pp. 165-169. [CrossRef]

33. Zhang, Q.; Saad, W.; Bennis, M.; Lu, X.; Debbah, M.; Zuo, W. Predictive Deployment of UAV Base Stations in Wireless Networks: Machine Learning Meets Contract Theory. IEEE Trans. Wirel. Commun. 2021, 20, 637-652. [CrossRef]

34. Alzenad, M.; El-Keyi, A.; Lagum, F.; Yanikomeroglu, H. 3-D Placement of an Unmanned Aerial Vehicle Base Station (UAV-BS) for Energy-Efficient Maximal Coverage. IEEE Wirel. Commun. Lett. 2017, 6, 434-437. [CrossRef]

35. Amponis, G.; Lagkas, T.; Sarigiannidis, P.; Vitsas, V.; Fouliras, P.; Wan, S. A survey on FANET routing from a cross-layer design perspective. J. Syst. Archit. 2021, 120, 102281. [CrossRef]

36. Nikam, V.B.; Arora, A.; Lambture, D.; Zaveri, J.; Shinde, P.; More, M. Optimal Positioning of Small Cells for Coverage and Cost Efficient 5G Network Deployment: A Smart Simulated Annealing Approach. In Proceedings of the 2020 IEEE 3rd 5G World Forum (5GWF), Bangalore, India, 10-12 September 2020; pp. 454-459. [CrossRef]

37. Hunukumbure, M.; Tsoukaneri, G. Cost Analysis for Drone Based 5G eMBB Provision to Emergency Services. In Proceedings of the 2019 IEEE Globecom Workshops (GC Wkshps), Waikoloa, HI, USA, 9-13 December 2019; pp. 1-5. [CrossRef]

38. Giordani, M.; Polese, M.; Mezzavilla, M.; Rangan, S.; Zorzi, M. Toward 6G Networks: Use Cases and Technologies. IEEE Commun. Mag. 2020, 58, 55-61. [CrossRef] 